PROCEEDINGS OF THE

AMERICAN MATHEMATICAL SOCIETY

Volume 128, Number 5, Pages 1391-1396

S 0002-9939(99)05159-X

Article electronically published on August 5, 1999

\title{
THE HAUSDORFF OPERATOR IS BOUNDED ON THE REAL HARDY SPACE $H^{1}(\mathbb{R})$
}

\author{
ELIJAH LIFLYAND AND FERENC MÓRICZ \\ (Communicated by Christopher D. Sogge)
}

\begin{abstract}
We prove that the Hausdorff operator generated by a function $\varphi \in L^{1}(\mathbb{R})$ is bounded on the real Hardy space $H^{1}(\mathbb{R})$. The proof is based on the closed graph theorem and on the fact that if a function $f$ in $L^{1}(\mathbb{R})$ is such that its Fourier transform $\widehat{f}(t)$ equals 0 for $t<0$ (or for $t>0$ ), then $f \in H^{1}(\mathbb{R})$.
\end{abstract}

\section{Preliminaries}

We recall that the Fourier transform $\widehat{f}$ of a function $f$ in $L^{1}(\mathbb{R})$ is defined by letting

$$
\widehat{f}(t):=\frac{1}{\sqrt{2 \pi}} \int_{\mathbb{R}} f(x) e^{-i t x} d x, \quad t \in \mathbb{R}
$$

while its Hilbert transform $\tilde{f}$ is defined by letting

$$
\widetilde{f}(x):=\frac{1}{\pi}(\mathrm{P} . \mathrm{V} .) \int_{0}^{\infty} \frac{f(x-u)-f(x+u)}{u} d u, \quad x \in \mathbb{R},
$$

where the principal value integral is defined to be $\lim _{\varepsilon \downarrow 0} \int_{\varepsilon}^{\infty}$. It is well known that this limit exists for almost every $x \in \mathbb{R}$.

The real Hardy space $H^{1}(\mathbb{R})$ is defined to be

$$
H^{1}(\mathbb{R}):=\left\{f \in L^{1}(\mathbb{R}): \tilde{f} \in L^{1}(\mathbb{R})\right\},
$$

endowed with the norm

$$
\|f\|_{H^{1}}:=\|f\|_{L^{1}}+\|\widetilde{f}\|_{L^{1}}
$$

where

$$
\|f\|_{L^{1}}:=\int_{\mathbb{R}}|f(x)| d x
$$

Received by the editors June 25, 1998.

1991 Mathematics Subject Classification. Primary 47B38; Secondary 46A30.

Key words and phrases. Fourier transform, Hilbert transform, real Hardy space $H^{1}(\mathbb{R})$, Hausdorff operator, Cesàro operator, closed graph theorem.

This research was partially supported by the Minerva Foundation through the Emmy Noether Institute at the Bar-Ilan University and by the Hungarian National Foundation for Scientific Research under Grant T 016393.

(C)2000 American Mathematical Society 
This space $H^{1}(\mathbb{R})$ is a Banach algebra under pointwise addition and scalar multiplication, and convolution. Furthermore, if $f \in H^{1}(\mathbb{R})$, then

$$
(\widetilde{f})^{\wedge}(t)=(-i \operatorname{sign} t) \widehat{f}(t), \quad t \in \mathbb{R}
$$

Hence it follows immediately that if $f \in H^{1}(\mathbb{R})$, then $\widehat{f}(0)=0$ and, by uniqueness of Fourier transform,

$$
(\widetilde{f})^{\sim}(t)=-f(t) \quad \text { a.e. }
$$

In particular, if $f \in H^{1}(\mathbb{R})$, then $\tilde{f} \in H^{1}(\mathbb{R})$ and

$$
\|\widetilde{f}\|_{H^{1}}=\|f\|_{H^{1}} \text {. }
$$

For more details, the reader is suggested to consult with [1], [5], [6].

\section{MAIN RESUlts}

We begin with the definition of the Hausdorff operator $\mathcal{H}$ acting on $L^{1}(\mathbb{R})$ generated by a function $\varphi$ belonging to $L^{1}(\mathbb{R})$. Given any function $f$ in $L^{1}(\mathbb{R}), \mathcal{H} f$ is defined to be that function in $L^{1}(\mathbb{R})$ for which

$$
(\mathcal{H} f)^{\wedge}(t)=\left(\mathcal{H}_{\varphi} f\right)^{\wedge}(t):=\int_{\mathbb{R}} \widehat{f}(t x) \varphi(x) d x, \quad t \in \mathbb{R} .
$$

The existence of such a function $\mathcal{H} f$ in $L^{1}(\mathbb{R})$ will be clear from the proof of Theorem 1 below.

The summability properties of $\mathcal{H} f(x)$ are well known as $x \rightarrow \infty$. (See, for example [4 pp. 275-278].) The objective here is to determine the Fourier analytic properties of $\mathcal{H} f$ on the class of functions in $L^{1}(\mathbb{R})$ and $H^{1}(\mathbb{R})$.

The following Theorem 1 is the special case of [2, Theorem 1] by Georgakis.

Theorem 1. If $\varphi \in L^{1}(\mathbb{R})$, then the Hausdorff operator $\mathcal{H}=\mathcal{H}_{\varphi}: L^{1}(\mathbb{R}) \rightarrow L^{1}(\mathbb{R})$ is bounded:

$$
\left\|\mathcal{H}_{\varphi}\right\|:=\sup _{\|f\|_{L^{1}} \leq 1}\left\|\mathcal{H}_{\varphi} f\right\|_{L^{1}} \leq\|\varphi\|_{L^{1}}
$$

Definition (2.1) applies to the particular case where $f \in H^{1}(\mathbb{R})$.

Theorem 2. If $\varphi \in L^{1}(\mathbb{R})$, then the Hausdorff operator $\mathcal{H}=\mathcal{H}_{\varphi}: H^{1}(\mathbb{R}) \rightarrow$ $H^{1}(\mathbb{R})$ is bounded.

Our last theorem expresses an interchangeability relation, under the assumption that the support of $\varphi$ is contained in one of the half-axes.

Theorem 3. Assume $\varphi \in L^{1}(\mathbb{R})$.

$$
\varphi(x)=0 \quad \text { for } \quad x<0,
$$

then we have

$$
(\mathcal{H} f)^{\sim}=\mathcal{H} \widetilde{f}, \quad f \in H^{1}(\mathbb{R})
$$

(ii) If

$$
\varphi(x)=0 \quad \text { for } \quad x>0
$$

then we have

$$
(\mathcal{H} f)^{\sim}=-\mathcal{H} \tilde{f}, \quad f \in H^{1}(\mathbb{R}) .
$$


As a corollary of Theorem 3, we may deduce the following: If $\varphi \in L^{1}(\mathbb{R})$, then

$$
\left(\mathcal{H}_{\varphi} f\right)^{\sim}=\mathcal{H}_{\operatorname{sign}(\cdot) \varphi(\cdot)} \tilde{f}, \quad f \in H^{1}(\mathbb{R}) .
$$

Remark. If $\varphi(x):=\chi_{(0,1)}(x)$, the indicator function of the unit interval $(0,1)$, then (2.1) is of the following form:

$$
(\mathcal{H} f)^{\wedge}(t):=\int_{0}^{1} \widehat{f}(t x) d x=\frac{1}{t} \int_{0}^{t} \widehat{f}(u) d u, \quad t \neq 0 .
$$

In this case, $\mathcal{H}$ is the well-known Cesàro operator, for which Theorems 2 and 3 were proved in [3].

In the capacity of $\varphi$ in Theorems 1-3, one may take a number of the usual summability kernels including

$$
e^{-|x|}, \quad e^{-x^{2} / 2}, \quad 1 /\left(1+x^{2}\right), \quad \text { etc. }
$$

or the "one-sided" versions of these, while multiplying them by one of the indicator functions $\chi_{(0, \infty)}(x)$ and $\chi_{(-\infty, 0)}(x)$.

\section{Proofs}

We begin with three auxiliary results.

Lemma 1. If $f$ and $\varphi$ both belong to $L^{1}(\mathbb{R})$, and $\mathcal{H} f$ is defined in $(2.1)$, then

$$
(\mathcal{H} f)^{\wedge}(t)=\frac{1}{|t|} \int_{\mathbb{R}} \widehat{f}(u) \varphi(u / t) d u, \quad t \neq 0,
$$

and

$$
(\mathcal{H} f)^{\wedge}(t)=\int_{\mathbb{R}} f(\xi) \widehat{\varphi}(t \xi) d \xi, \quad t \in \mathbb{R} .
$$

Proof. It is routine: integrate by substitution and use Fubini's theorem.

Lemma 2. If $f \in L^{1}(\mathbb{R})$ is such that $\widehat{f}(t)=0$ for $t<0$, then $f \in H^{1}(\mathbb{R})$.

The symmetric counterpart of Lemma 2 says that if $f \in L^{1}(\mathbb{R})$ is such that $\widehat{f}(t)=0$ for $t>0$, then $f \in H^{1}(\mathbb{R})$.

Lemma 3. If $f \in H^{1}(\mathbb{R})$, then there exist two functions $f_{1}$ and $f_{2}$, both in $H^{1}(\mathbb{R})$ such that $f=f_{1}+f_{2}$,

$$
\widehat{f}_{1}(t)=0 \quad \text { for } \quad t<0, \quad \text { and } \quad \widehat{f}_{2}(t)=0 \text { for } t>0 .
$$

Lemmas 2 and 3 are well known. (See, for example, [3].)

As we have noted Theorem 1 follows from [2, Theorem 1] by Georgakis. For the reader's convenience, we present here a direct proof.

Proof of Theorem 1. We claim that the function $\mathcal{H} f$ defined by

$$
\mathcal{H} f(x):=\int_{\mathbb{R}} \frac{f(\xi)}{|\xi|} \varphi\left(\frac{x}{\xi}\right) d \xi, \quad x \in \mathbb{R},
$$

is in $L^{1}(\mathbb{R})$ and satisfies $(2.1)$. 
In fact, by Fubini's theorem,

$$
\begin{aligned}
\int_{\mathbb{R}}|\mathcal{H} f(x)| d x & \leq \int_{\mathbb{R}} d x \int_{\mathbb{R}}\left|\frac{f(\xi)}{\xi}\right|\left|\varphi\left(\frac{x}{\xi}\right)\right| d \xi \\
& =\int_{0}^{\infty} \frac{|f(\xi)|}{\xi} d \xi \int_{\mathbb{R}}\left|\varphi\left(\frac{x}{\xi}\right)\right| d x-\int_{-\infty}^{0} \frac{|f(\xi)|}{\xi} d \xi \int_{\mathbb{R}}\left|\varphi\left(\frac{x}{\xi}\right)\right| d x \\
& =\int_{\mathbb{R}}|f(\xi)| d \xi \int_{\mathbb{R}}|\varphi(u)| d u<\infty,
\end{aligned}
$$

which proves (2.2), as well.

By (3.3) and Fubini's theorem,

$$
\begin{aligned}
(\mathcal{H} f)^{\wedge}(t)= & \frac{1}{\sqrt{2 \pi}} \int_{\mathbb{R}} e^{-i x t} d x \int_{\mathbb{R}} \frac{f(\xi)}{|\xi|} \varphi\left(\frac{x}{\xi}\right) d \xi \\
= & \int_{0}^{\infty} \frac{f(\xi)}{\xi} d \xi \frac{1}{\sqrt{2 \pi}} \int_{\mathbb{R}} \varphi\left(\frac{x}{\xi}\right) e^{-i t x} d x \\
& -\int_{-\infty}^{0} \frac{f(\xi)}{\xi} d \xi \frac{1}{\sqrt{2 \pi}} \int_{\mathbb{R}} \varphi\left(\frac{x}{\xi}\right) e^{-i t x} d x \\
= & \int_{\mathbb{R}} f(\xi) d \xi \frac{1}{\sqrt{2 \pi}} \int_{\mathbb{R}} \varphi(u) e^{-i t \xi u} d u,
\end{aligned}
$$

which is (2.1), due to (3.2).

Proof of Theorem 2. First, we assume that $\varphi \in L^{1}(\mathbb{R})$ is such that condition (2.3) is satisfied. Given $f \in H^{1}(\mathbb{R})$, we consider the decomposition $f=f_{1}+f_{2}$ provided by Lemma 3. The Hausdorff operator is clearly linear:

$$
\mathcal{H} f=\mathcal{H} f_{1}+\mathcal{H} f_{2} .
$$

By Lemma $3, \widehat{f}_{1}(u)=0$ for $u<0$; and by $(2.3), \varphi(u / t)=0$ for $u>0$ and $t<0$. Thus, we have

$$
\left(\mathcal{H} f_{1}\right)^{\wedge}(t)=\frac{1}{|t|} \int_{0}^{\infty} \widehat{f}_{1}(u) \varphi(u / t) d u=0 \quad \text { for } \quad t<0 .
$$

In a similar manner, we find

$$
\left(\mathcal{H} f_{2}\right)^{\wedge}(t)=\frac{1}{|t|} \int_{-\infty}^{0} \widehat{f_{2}}(u) \varphi(u / t) d u=0 \quad \text { for } \quad t>0 .
$$

By Theorem 1 and Lemma 2, both $\mathcal{H} f_{1}$ and $\mathcal{H} f_{2}$ belong to $H^{1}(\mathbb{R})$. From (3.4) it follows that $\mathcal{H} f \in H^{1}(\mathbb{R})$.

It is plain that the above argument works in the other particular case where, instead of (2.3), we have (2.5).

In the general case, we decompose $\varphi \in L^{1}(\mathbb{R})$ in the trivial way:

$$
\varphi(x)=\varphi_{1}(x)+\varphi_{2}(x),
$$

where

$$
\varphi_{1}(x):=\varphi(x) \chi_{(0, \infty)}(x) \quad \text { and } \quad \varphi_{2}(x):=\varphi(x)-\varphi_{1}(x) ;
$$

$\chi_{(0, \infty)}(x)$ being the indicator function of the half-axis $(0, \infty)$. Clearly, we have

$$
\mathcal{H}_{\varphi} f=\mathcal{H}_{\varphi_{1}} f+\mathcal{H}_{\varphi_{2}} f, \quad f \in H^{1}(\mathbb{R}),
$$


and the above particular cases apply separately to $\mathcal{H}_{\varphi_{1}}$ and $\mathcal{H}_{\varphi_{2}}$, respectively. Thus, we have justified our claim that $\mathcal{H} f \in H^{1}(\mathbb{R})$ whenever $f \in H^{1}(\mathbb{R})$.

Now, we shall apply the closed graph theorem, which says that a linear operator mapping a Banach space into another Banach space is bounded if and only if it is closed. We note that the closed graph theorem holds true under more general conditions, but the above formulation is enough for our purposes.

Obviously, the Hausdorff operator $\mathcal{H}$ is linear. It remains to check that $\mathcal{H}$ is closed. To this effect, assume that a sequence $\left\{f_{n}: n=1,2, \ldots\right\}$ is given in $H^{1}(\mathbb{R})$ such that

$$
f_{n} \rightarrow f \quad \text { and } \quad \mathcal{H} f_{n} \rightarrow g \quad \text { as } \quad n \rightarrow \infty,
$$

with some $f$ and $g$ both in $H^{1}(\mathbb{R})$, convergence being meant in the norm (1.1) of $H^{1}(\mathbb{R})$ in both cases. It is plain that the limits in (3.5) hold in the norm of $L^{1}(\mathbb{R})$, as well. By Theorem 1, the Hausdorff operator $\mathcal{H}$ is closed in $L^{1}(\mathbb{R})$, whence we conclude that $\mathcal{H} f=g$. This means that the operator $\mathcal{H}$ is closed in $H^{1}(\mathbb{R})$, too.

Proof of Theorem 3. First, we set $g:=\mathcal{H} \tilde{f}$. By definition and (1.2), we have

$$
\begin{aligned}
\widehat{g}(t):=\int_{\mathbb{R}}(\widetilde{f})^{\wedge}(t x) \varphi(x) d x=\int_{\mathbb{R}}(-i \operatorname{sign} t x) \widehat{f}(t x) \varphi(x) d x \\
\quad=(-i \operatorname{sign} t) \int_{0}^{\infty} \widehat{f}(t x) \varphi(x) d x+(i \operatorname{sign} t) \int_{-\infty}^{0} \widehat{f}(t x) \varphi(x) d x, \quad t \in \mathbb{R} .
\end{aligned}
$$

Second, we set $h:=(\mathcal{H} f)^{\sim}$. By (1.3), this is equivalent to the following: $\mathcal{H} f=$ $-\widetilde{h}$. By $(1.2)$,

$$
(\mathcal{H} f)^{\wedge}(t)=(-\widetilde{h})^{\wedge}(t)=(i \operatorname{sign} t) \widehat{h}(t)
$$

whence

$$
\begin{aligned}
\widehat{h}(t) & =(-i \operatorname{sign} t)(\mathcal{H} f)^{\wedge}(t) \\
& :=(-i \operatorname{sign} t) \int_{\mathbb{R}} \widehat{f}(t x) \varphi(x) d x, \quad t \in \mathbb{R} .
\end{aligned}
$$

Comparing (3.6) and (3.7), and taking into account (2.3) yields

$$
\widehat{g}(t)=\widehat{h}(t), \quad t \in \mathbb{R} ;
$$

while taking into account $(2.5)$ yields

$$
\widehat{g}(t):=-\widehat{h}(t), \quad t \in \mathbb{R} .
$$

By uniqueness of the Fourier transform, we conclude (2.4) and (2.6), respectively.

\section{REFERENCES}

1. R. E. Edwards, Fourier series. A modern introduction, Holt, Rinehart and Winston, New York, 1967. MR 35:7062

2. C. Georgakis, The Hausdorff mean of a Fourier-Stieltjes transform, Proc. Amer. Math. Soc. 116 (1992), 465-471. [MR 92m:42009]

3. D. V. Giang and F. Móricz, The Cesàro operator is bounded on the Hardy space $H^{1}$, Acta Sci. Math. (Szeged) 61 (1995), 535-544. MR 96m:47051 
4. G. H. Hardy, Divergent series, Clarendon, Oxford, 1949. |MR 11:25a

5. E. M. Stein and G. Weiss, Introduction to Fourier analysis on Euclidean spaces, Princeton Univ. Press, New Jersey, 1971. MR 46:4102

6. E. C. Titchmarsh, Introduction to the theory of Fourier integrals, Clarendon, Oxford, 1937.

Department of Mathematics and Computer Science, Bar-ilan University, 52900 RamatGAN, ISRAEL

E-mail address: liflyand@macs.biu.ac.il

Bolyai Institute, University of Szeged, Aradi Vértanúk tere 1, 6720 Szeged, Hungary

E-mail address: moricz@math.u-szeged.hu 